\title{
A Filterless Frequency 32-Tupling Photonic Scheme To Generate Sub-Terahertz Wave Signal Enabled By Optical Polarization Modulators
}

Xinqiao Chen ( $\sim$ chenxinqiao9999@163.com )

Communication University of China https://orcid.org/0000-0001-8522-3227

Xiaorui Liu

Communication University of China

Zhihan Li

Communication University of China

\section{Research Article}

Keywords: Polarization modulator, Sub-Terahertz wave, OSSR, RFSSR

Posted Date: August 30th, 2021

DOI: https://doi.org/10.21203/rs.3.rs-767668/v1

License: (c) (i) This work is licensed under a Creative Commons Attribution 4.0 International License.

Read Full License 


\title{
A filterless frequency 32-tupling photonic scheme to generate Sub-Terahertz wave signal enabled by optical polarization modulators
}

\author{
Xinqiao Chen* · Xiaorui Liu • Zhihan Li
}

Received: date / Accepted: date

\begin{abstract}
A novel scheme based on polarization modulators to generate SubTerahertz wave (Sub-THz) signal with frequency 32-tupling is proposed. The system consists of two subsystems in cascade, and each subsystem consists of 4 paralleled polarization modulators. In this scheme, by properly controlling two subsystems, an optical signal with optical carrier and \pm 16 th-order optical sidebands is achieved. Next, by adjusting the optical attenuator and optical phase shifter, the optical carrier can be canceled. Finally, the Sub-THz wave with frequency 32 -tupling can be obtained after beaten in the photodetector. The results show the \pm 16 th-order optical sideband suppression ratio (OSSR) is derived as $55.7 \mathrm{~dB}$ and the radio frequency sideband suppression ratio (RFSSR) of the 32-tupling $\mathrm{Sub}-\mathrm{THz}$ wave is derived as $43.66 \mathrm{~dB}$, which are consistent with the theoretical analysis very well. Meanwhile, the effects of phase offset, modulation index, and attenuator deviation on suppression ratio are analyzed.
\end{abstract}

Keywords Polarization modulator · Sub-Terahertz wave · OSSR · RFSSR

Mathematics Subject Classification (2020) MSC code1 $\cdot$ MSC code $2 \cdot \operatorname{more}$

\section{Introduction}

To meet the increasing demand of mobile communication and multimedia services, higher capacity wireless access network is necessary. Sub-Terahertz carrier frequency is a potential solution for growing data demands. The first IEEE standard over Sub-Terahertz (specifically, 253-322 GHz), IEEE std. 802.15.3d -2017, primarily illustrates the feasibility of Sub-THz communications[1, 2]. Compared with the conventional technology in electrical domain, the optical generation scheme for millimeter wave (mm-wave) is desirable, since it can produce signal with higher frequency, wider bandwidth, and anti-electromagnetic interference[3]. Therefore, the optical generation of high frequency mm-wave or Sub-Terahertz wave has aroused much interest in recent years.

School of Information and Communication Engineering, Communication University of China, Beijing 100024, China

* Corresponding author E-mail: chenxinqiao9999@163.com 
Many photonic schemes based on external modulation to generate mm-wave draw great attention, due to its stable operation, low complexity and cost effective [4]. In order to eliminate the harmonic sidebands which will induce fiber dispersion, some wavelength selection devices are used in these schemes, such as a wavelengthfixed optical notch filter [5], optical interleavers [6, 7], or fiber Bragg gratings [8]. Nevertheless, the frequency tunability is limited for the fixed bandwidth of these devices. To solve the challenging problem caused by restricted frequency tunability, the filterless methods to produce mm-wave are preferred. There have been many demonstrations on the filterless generation of mm-wave based on Mach-Zehnder Modulator (MZM) in parallel [9], or in cascade [10]. Moreover, Wang et al. have proposed several schemes to generate vector mm-wave based on MZM and digital modulation format combinations. Compared with conventional mm-wave signals, vector mm-wave can simultaneously load signals containing amplitude information and phase information, thus improving the spectrum efficiency of the system [1113].

However, the generation of high-frequency mm-wave based on MZM has a problem of offset drift, which requires a complex offset control circuit. Because optical generation mm-wave by polarization modulator (PolM) does not require DC bias, the system shows better stability [14]. Zhu et al. have proposed a common approach to generate frequency octupling, sextupling, or quadrupling mm-wave by two PolMs [15]. Abouelez et al. have proposed a scheme to generate a frequency 12tupling mm-wave by cascaded dual parallel polarization modulator (DP-PolM) [16]. Kumar et al. have proposed a method to generate frequency 24-tupling mm-wave signal by optical filters and SOA, but the usage of optical filter limits the frequency tuning range [17]. Chaudhuri et al. have proposed a plan to generate mm-wave with frequency 24-tupling by two DP-PolMs[18]. The generated mm-wave has an OSSR and RFSSR of about $34.4 \mathrm{~dB}$ and $25.8 \mathrm{~dB}$.

In this paper, a filterless photonic scheme to generate Sub-Terahertz wave signal with frequency 32-tupling is proposed and demonstrated. By properly controlling the two subsystems, optical signals with mainly 16th-order sidebands and carrier component are achieved. Then, by tuning the optical attenuator and optical phase shifter, the optical carrier can be canceled. Finally, the frequency 32-tupling Sub-Terahertz wave is generated after the photodetector beating.

\section{System design and principle}

The schematic diagram of generating frequency 32-tupling Sub-THz wave based on polarization modulators is shown in Fig. 1. The system consists of two subsystems (i.e., Sub A and Sub B) in cascade, and each subsystem consists of 4 paralleled polarization modulators and a polarizer ( $\mathrm{Pol})$. A PolM is made up of a polarization beam splitter, two phase modulators, and a polarization beam combiner[16]. The PolM is a special phase modulator that supports both TE and TM modes with opposite phase modulation indexes. The joint operation of a PolM, a PC (polarization controller), and a polarizer is equivalent to an MZM, with the bias point being controlled by adjusting the phase difference between the two orthogonal modes via tuning the PC. 


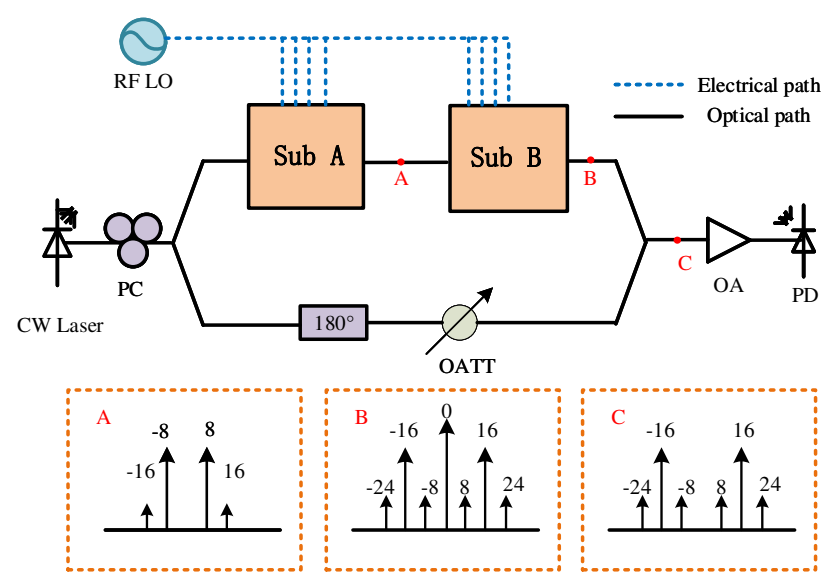

Fig. 1: Schematic diagram of generating frequency 32-tupling Sub-THz wave based on polarization modulators. CW Laser: continuous wave laser; PC: polarization controller; RF LO: radio frequency local oscillator; OATT: optical attenuator; OA: optical amplifier; PD: photodiode.

The optical carrier emitted from the continuous wave laser (CW Laser) is assumed as

$$
E_{\text {in }}=E_{\mathrm{c}} \exp \left(\mathrm{j} \omega_{\mathrm{c}} t\right)
$$

where $E_{\mathrm{c}}$ and $\omega_{\mathrm{c}}$ are amplitude and angular frequency of the optical carrier, respectively. The optical carrier is then converted into a linearly polarized light by $\mathrm{PC}$ with an angle of $45^{\circ}$ between the polarization direction and the transmission axis. The optical wave is divided into two beams by a $1 \times 2$ power splitter. One is sent into the cascaded system of Sub A and Sub B, and the other passes through the optical phase shifter and the optical attenuator.

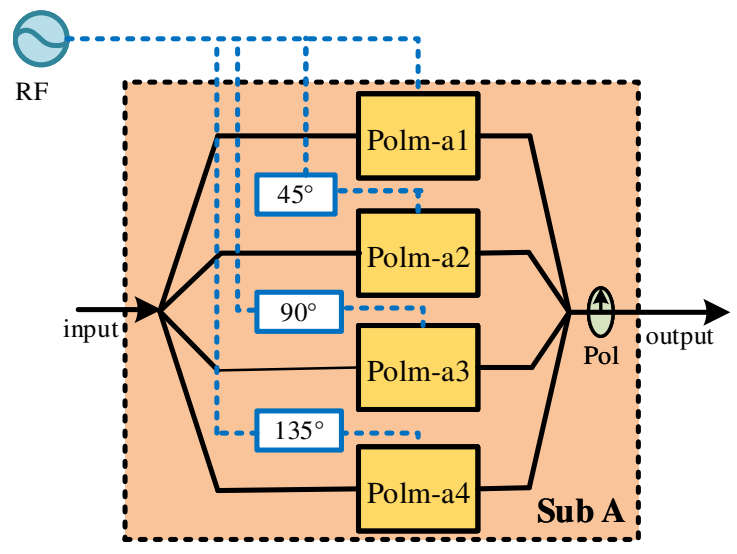

Fig. 2: schematic diagram of Sub A 
As shown in Fig. 2, the input optical signal is divided into 4 beams by a $1 \times 4$ power splitter in Sub A. Afterwards, the 4 branches are severally injected into four PolMs in parallel, named PolM-ai $(\mathrm{i}=1,2,3,4)$, respectively. The RF driving signal applied to PolM-ai is defined as

$$
V(t)=V_{\mathrm{RF}} \sin \left(\omega_{\mathrm{RF}} t+(i-1) \Delta \varphi\right)
$$

where $V_{\mathrm{RF}}$ and $\omega_{\mathrm{RF}}$ are amplitude and angular frequency of the RF driving signal, $\Delta \varphi$ is the phase difference of the driving signal between two adjacent PolM-ai. Thus, the output of each PolM-ai can be expressed as

$$
\begin{aligned}
{\left[\begin{array}{l}
E_{i x} \\
E_{i y}
\end{array}\right] } & =\frac{1}{8} E_{\mathrm{in}}\left[\begin{array}{c}
\cos 45^{\circ} \exp \left(\mathrm{j} m \cos \left(\omega_{\mathrm{RF}} t+(i-1) \Delta \varphi\right)\right) \\
\sin 45^{\circ} \exp \left(-\mathrm{j} m \cos \left(\omega_{\mathrm{RF}} t+(i-1) \Delta \varphi\right)\right)
\end{array}\right] \\
& =\frac{\sqrt{2}}{16} E_{\mathrm{c}} \exp \left(j \omega_{\mathrm{c}} t\right)\left[\begin{array}{c}
\exp \left(\mathrm{j} m \cos \left(\omega_{\mathrm{RF}} t+(i-1) \Delta \varphi\right)\right) \\
\exp \left(-\mathrm{j} m \cos \left(\omega_{\mathrm{RF}} t+(i-1) \Delta \varphi\right)\right)
\end{array}\right]
\end{aligned}
$$

where $m$ is the modulation index of PolM and it is equal to $\pi V_{\mathrm{RF}} / V_{\pi} . V_{\pi}$ is the half-wave voltage. Consequently, the output signal after the $4 \times 1$ power combiner can be given by

$$
\begin{aligned}
E_{i} & =\sum_{i=1}^{4}\left[\begin{array}{l}
E_{i x} \\
E_{i y}
\end{array}\right] \\
& =\frac{\sqrt{2}}{16} E_{\mathrm{c}} \exp \left(j \omega_{\mathrm{c}} t\right) \sum_{i=1}^{4}\left[\begin{array}{c}
\exp \left(\mathrm{j} m \cos \left(\omega_{\mathrm{RF}} t+(i-1) \Delta \varphi\right)\right) \\
\exp \left(-\mathrm{j} m \cos \left(\omega_{\mathrm{RF}} t+(i-1) \Delta \varphi\right)\right)
\end{array}\right]
\end{aligned}
$$

As Pol has an orientation of $45^{\circ}$ to the transmission axis, its output is expressed as

$$
\begin{aligned}
E_{\mathrm{A}}= & \cos 45^{\circ} \sum_{i=1}^{4} E_{i x}+\sin 45^{\circ} \sum_{i=1}^{4} E_{i y} \\
= & \frac{1}{16} E_{\mathrm{c}} \exp \left(\mathrm{j} \omega_{\mathrm{c}} t\right) \sum_{i=1}^{4} \exp \left\{j m \cos \left[\omega_{\mathrm{RF}} t+(i-1) \Delta \varphi\right]\right\} \\
& +\frac{1}{16} E_{\mathrm{c}} \exp \left(\mathrm{j} \omega_{\mathrm{c}} t\right) \sum_{i=1}^{4} \exp \left\{-j m \cos \left[\omega_{\mathrm{RF}} t+(i-1) \Delta \varphi\right]\right\} \\
= & \frac{1}{8} E_{\mathrm{c}} \exp \left(\mathrm{j} \omega_{\mathrm{c}} t\right) \sum_{i=1}^{4} \cos \left\{m \cos \left[\omega_{\mathrm{RF}} t+(i-1) \Delta \varphi\right]\right\}
\end{aligned}
$$

According to Eq. (5), the output optical signal of the polarizer has been converted from a phase modulation signal to an intensity modulation signal, where the amplitude modulation factor is $\cos \left\{m \cos \left[\omega_{\mathrm{RF}} t+(i-1)\right] \Delta \varphi\right\}$.

Using the Jacobi-Anger formula, Eq. (5) can be expanded as

$$
\begin{aligned}
& E_{\mathrm{A}}=\frac{1}{8} E_{\mathrm{c}} \exp \left(\mathrm{j} \omega_{\mathrm{c}} t\right)\left\{\begin{array}{l}
4 \mathrm{~J}_{0}(m)+ \\
\sum_{i=1}^{4}\left[2 \sum_{n=1}^{\infty}(-1)^{n} \mathrm{~J}_{2 n}(m) \cos \left(2 n \omega_{\mathrm{RF}} t+2 n(i-1) \pi / 4\right)\right]
\end{array}\right. \\
& =\frac{1}{2} E_{\mathrm{c}} \exp \left(\mathrm{j} \omega_{\mathrm{c}} t\right)\left[\mathrm{J}_{0}(m)+2 \mathrm{~J}_{8}(m) \cos \left(8 \omega_{\mathrm{RF}} t\right)+2 \mathrm{~J}_{16}(m) \cos \left(16 \omega_{\mathrm{RF}} t\right) \ldots\right]
\end{aligned}
$$


where $\mathrm{J}_{n}(m)$ denotes the Bessel function of the first kind of nth-order.

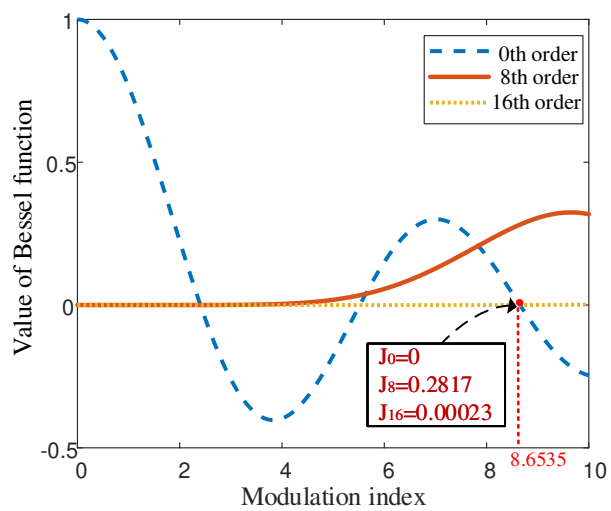

Fig. 3: Graph of the first-class Bessel function of 0th-order, 8th-order, and 16thorder

Eq. (6) shows that 8 nth-order optical sidebands have been retained in the output optical signal of sub A. Graph of the first-class Bessel function of 0thorder, 8th-order, and 16th-order is shown in Fig. 3. As demonstrated, when the modulation index $\mathrm{m}$ is equal to 8.6535 , the values of $\mathrm{J}_{0}(m), \mathrm{J}_{8}(m)$, and $\mathrm{J}_{16}(m)$ are $0,0.2817$, and $2.3 \times 10-4$, separately. As a result, when m equals 8.6535 , the optical carrier is suppressed, and the optical sideband signals above 16th-order are small and can be ignored.

The output signal from Sub A can be written as

$$
E_{\mathrm{A}}=E_{\mathrm{c}} \exp \left(\mathrm{j} \omega_{\mathrm{c}} t\right)\left[\mathrm{J}_{8}(m) \cos \left(8 \omega_{\mathrm{RF}} t\right)+\mathrm{J}_{16}(m) \cos \left(16 \omega_{\mathrm{RF}} t\right)\right]
$$

Sub B's structure is the same as Sub A's. Substituting for EA and expanding, the output of Sub B can be expressed as

$$
\begin{aligned}
E_{\mathrm{B}}= & 2 E_{\mathrm{A}} \odot\left[\mathrm{J}_{8}(m) \cos \left(8 \omega_{\mathrm{RF}} t\right)+\mathrm{J}_{16}(m) \cos \left(16 \omega_{\mathrm{RF}} t\right)\right] \\
= & 2 E_{\mathrm{c}} \exp \left(\mathrm{j} \omega_{\mathrm{c}} t\right)\left[\mathrm{J}_{8}(m) \cos \left(8 \omega_{\mathrm{RF}} t\right)+\mathrm{J}_{16}(m) \cos \left(16 \omega_{\mathrm{RF}} t\right)\right] \\
& \odot\left[\mathrm{J}_{8}(m) \cos \left(8 \omega_{\mathrm{RF}} t\right)+\mathrm{J}_{16}(m) \cos \left(16 \omega_{\mathrm{RF}} t\right)\right] \\
= & E_{\mathrm{c}} \exp \left(\mathrm{j} \omega_{\mathrm{c}} t\right)\left\{\mathrm{J}_{8}^{2}(m)+\mathrm{J}_{16}{ }^{2}(m)+2 \mathrm{~J}_{8}(m) \mathrm{J}_{16}(m) \cos \left(8 \omega_{\mathrm{RF}} t\right)\right. \\
& +\mathrm{J}_{8}^{2}(m) \cos \left(16 \omega_{\mathrm{RF}} t\right)+2 \mathrm{~J}_{8}(m) \mathrm{J}_{16}(m) \cos \left(24 \omega_{\mathrm{RF}} t\right) \\
& \left.+\mathrm{J}_{16}{ }^{2}(m) \cos \left(32 \omega_{\mathrm{RF}} t\right)\right\}
\end{aligned}
$$

Eq. (8) shows that a new harmonic optical signal is generated at the center frequency, which is also the maximum harmonic optical signal.

The output optical signal of sub B combines with the optic signal from the attenuator by a $2 \times 1$ power combiner, and the carrier component can be canceled[19]. In order to cancel the carrier component, the value of the optical phase shifter is set to be $180^{\circ}$, and the attenuation of the optical attenuator is adjusted to be

$$
\alpha=20 \log _{10}\left\{2\left[\mathrm{~J}_{8}^{2}(8.6535)+\mathrm{J}_{16}{ }^{2}(8.6535)\right]\right\}^{-1}=15.985 \mathrm{~dB}
$$


where $\alpha$ is the attenuation value. The output signal of the power combiner, as illustrated in Fig. 1(C), can be simplified as

$$
\begin{aligned}
E_{\mathrm{D}}(t)= & E_{\mathrm{c}} \exp \left(\mathrm{j} \omega_{\mathrm{c}} t\right)\left\{2 \mathrm{~J}_{8}(m) \mathrm{J}_{16}(m) \cos \left(8 \omega_{\mathrm{RF}} t\right)+\mathrm{J}_{8}^{2}(m) \cos \left(16 \omega_{\mathrm{RF}} t\right)\right. \\
& \left.+2 \mathrm{~J}_{8}(m) \mathrm{J}_{16}(m) \cos \left(24 \omega_{\mathrm{RF}} t\right)+\mathrm{J}_{16}^{2}(m) \cos \left(32 \omega_{\mathrm{RF}} t\right)\right\}
\end{aligned}
$$

After the square-law detection using a photodetector, ignoring higher-order optical sidebands greater than $32 \omega_{\mathrm{RF}}$, the photocurrent output from the photodetector $(\mathrm{PD})$ is described by

$$
\begin{aligned}
I_{\mathrm{PD}}(t) \propto & \Re E_{D} \times E_{D}^{*} \\
= & \Re E_{\mathrm{c}}^{2}\left\{\left[4 \mathrm{~J}_{8}^{2}(m) \mathrm{J}_{16}^{2}(m)+\mathrm{J}_{8}^{4}(m) / 2\right]\right. \\
& +4 \mathrm{~J}_{8}^{3}(m) \mathrm{J}_{16}(m) \cos \left(8 \omega_{\mathrm{RF}} t\right)+6 \mathrm{~J}_{8}^{2}(m) \mathrm{J}_{16}^{2}(m) \cos \left(16 \omega_{\mathrm{RF}} t\right) \\
& +2 \mathrm{~J}_{8}^{3}(m) \mathrm{J}_{16}(m) \cos \left(24 \omega_{\mathrm{RF}} t\right) \\
& +\left[4 \mathrm{~J}_{8}^{2}(m) \mathrm{J}_{16}^{2}(m)+\mathrm{J}_{8}^{4}(m) / 2\right] \cos \left(32 \omega_{\mathrm{RF}} t\right) \\
& \left.+2 \mathrm{~J}_{8}^{3}(m) \mathrm{J}_{16}(m) \cos \left(40 \omega_{\mathrm{RF}} t\right)+2 \mathrm{~J}_{8}^{2}(m) \mathrm{J}_{16}^{2}(m) \cos \left(48 \omega_{\mathrm{RF}} t\right)\right\}
\end{aligned}
$$

where $\mathfrak{R}$ is the responsivity of the photodetector. It can be seen from Eq. (11) that the system generates the 32-tupling frequency millimeter wave.

From Eq. (10), we can see that the 8th-order optical sideband signal has the largest amplitude in harmonic component. According to the definition of optical sideband suppression ratio (OSSR), it can be given by

$$
\text { OSSR } \approx 10 \log _{10}\left[\frac{\mathrm{J}_{8}^{2}(8.6535)}{2 \mathrm{~J}_{16}(8.6535) \mathrm{J}_{8}(8.6535)}\right]^{2}=55.7 \mathrm{~dB}
$$

Besides, radio frequency sideband suppression ratio (RFSSR) is described as

$$
\operatorname{RFSSR} \approx 10 \log _{10}\left[\frac{4 \mathrm{~J}_{8}^{2}(8.6535) \mathrm{J}_{16}^{2}(8.6535)+\mathrm{J}_{8}^{4}(8.6535) / 2}{4 \mathrm{~J}_{8}^{3}(8.6535) \mathrm{J}_{16}(8.6535)}\right]^{2}=43.66 \mathrm{~dB}
$$

\section{Simulation results}

The simulation system of generating frequency 32-tupling Sub-THz wave by PolMs in cascade is designed by OptiSystem according to Fig. 1. The main parameters in simulation are set in Table 1.

Fig. 4(a) is the optical spectrum at point A in Fig. 1, which is the output of sub A. It can be seen that \pm 8 -order and \pm 16 -order optical sidebands are the main optical sideband. These optical sidebands above 16th-order can be ignored. From Fig. 4(b) is the optical spectrum at point B, we can see that the main optical component are the carrier and \pm 16 th-order optical sidebands in the Sub B. The \pm 8 th-order and \pm 24 th-order optical sidebands are small and can be ignored. Fig. 4 (c) is the optical spectrum at point $\mathrm{C}$, which is the composite optical signal from combiner. As shown in Fig. 4(c), the carrier component and harmonic optical sidebands are well suppressed. The 16th-order sidebands' power is $53.38 \mathrm{~dB}$ higher 
Table 1: Main parameters involved in simulation

\begin{tabular}{ll}
\hline Parameters Involved & Values \\
\hline Central frequency of CW Laser & $193.1 \mathrm{THz}$ \\
Linewidth of CW Laser & $10 \mathrm{MHz}$ \\
Power of CW Laser & $10 \mathrm{dBm}$ \\
Local Oscillator frequency & $10 \mathrm{GHz}$ \\
Gain of Amplifier & $20 \mathrm{~dB}$ \\
Noise figure of Amplifier & $4 \mathrm{~dB}$ \\
Responsivity of Photodiode & $0.8 \mathrm{~A} / \mathrm{W}$ \\
Dark current of Photodiode & $10 \mathrm{nA}$ \\
\hline
\end{tabular}

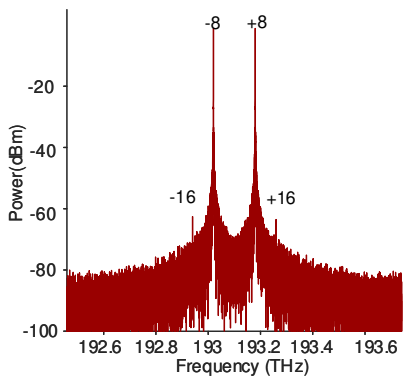

(a)

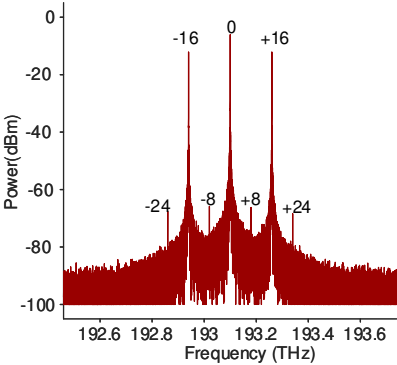

(b)

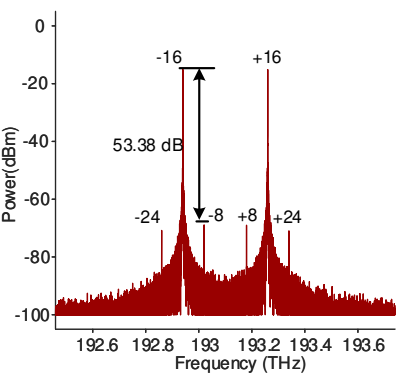

(c)

Fig. 4: output optical spectrum at (a) point $\mathrm{A}$; (b) point $\mathrm{B} ;(\mathrm{C})$ point $\mathrm{C}$

than that of the 8th-order's. It is consistent with the theoretical calculation of $55.7 \mathrm{~dB}$.

Fig. 5 is the electrical signal spectrum obtained by photodetector beating. It shows that the $\mathrm{Sub}-\mathrm{THz}$ wave signal desired has been generated at $320 \mathrm{GHz}$ frequency, which is 32 times of the input $10 \mathrm{GHz} R F$ driving signal. It is generated by beating \pm 16 th-order optical sidebands in $\mathrm{PD}$. There is also a visible harmonic mm-wave at $80 \mathrm{GHz}$ frequency. However, the $320 \mathrm{GHz}$ frequency signal's power is $42 \mathrm{~dB}$ higher than the harmonic mm-wave. It is in good accordance with the theoretical calculation of $43.66 \mathrm{~dB}$ above.

From the previous theoretical analysis, it is evident that the initial phase value of the RF driving signal, the modulation index of PolMs, and the attenuation of attenuator impact the purity of frequency 32-tupling Sub-THz wave signal. Thus, it is necessary to study the effect on the Sub-THz wave's purity, when these parameters deviate from the theoretical value above.

\section{1 effect on the OSSR and RFSSR by the offset of RF driving signal phase}

Fig. 6 shows the spectrum of the synthesized optical signal at Point $\mathrm{C}$ and the output spectrum at $\mathrm{PD}$ when $\mathrm{RF}$ driving phase deviates by $5^{\circ}$. As the phase deviates from the theoretical value, the odd order sideband cannot be well suppressed. The residual optical sideband is the main factor that leads to the decrease of signal performance. It can be seen from Fig. 6(a) that the power of the 16th-order optical 


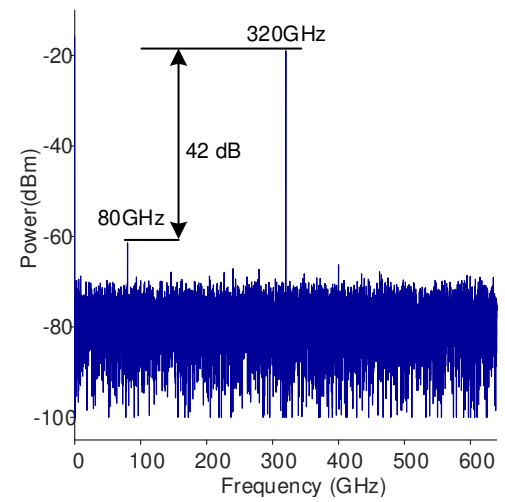

Fig. 5: Spectrum of electrical signal from photodetector

sideband is $18.3 \mathrm{~dB}$ higher than the maximum residual band. As the power of the unexpected optical sideband becomes larger, the power of the harmonic sidebands in the electrical signal increases greatly, as shown in Fig. 6(b).

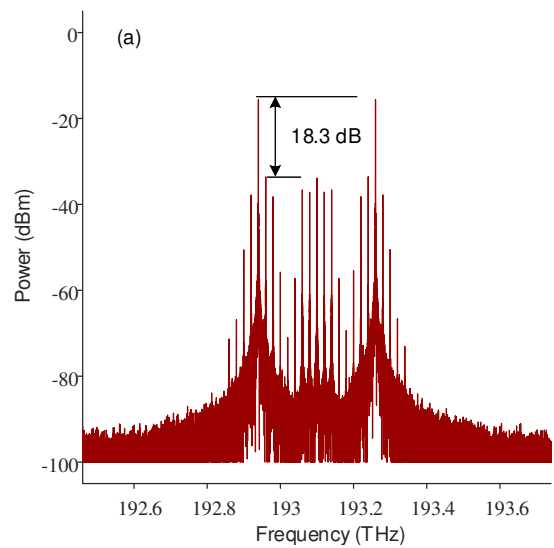

(a)

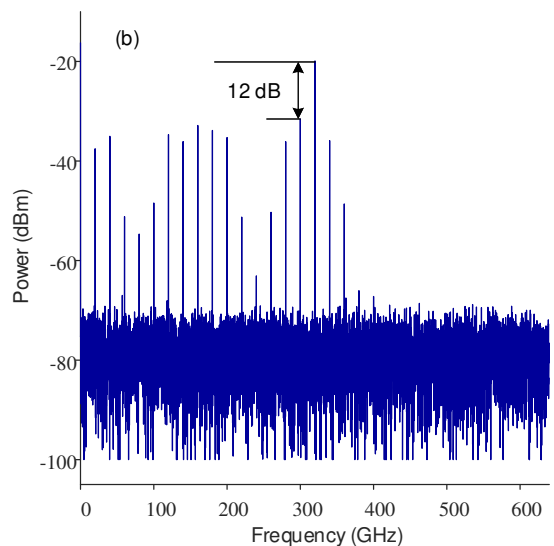

(b)

Fig. 6: Optical Spectrum and electrical spectrum when the phase offset is $5^{\circ}$

To analyze the influence of the initial phase of the RF driving signal, the PolMa1 is selected as $\pm 5^{\circ}$ deviations from theoretical value of $0^{\circ}$. As shown in Fig. 7, the OSSR and RFSSR of the optical signal and Sub-THz wave signal vary with the phase deviations. It indicates that when the phase deviation reaches $\pm 5^{\circ}$, the values of OSSR and RFSSR greatly drop from $53.38 \mathrm{~dB}$ to $18.36 \mathrm{~dB}$, and RFSSR drops from $42 \mathrm{~dB}$ to around $12 \mathrm{~dB}$. This is because when the initial phase deviates from its theoretical value, the different optical sidebands cannot be completely canceled out, resulting in a substantial increase in harmonic sidebands power and a decrease in rejection ratio. However, as long as the phase deviations are within 
the range of about $\pm 3^{\circ}$, the suppression ratio will be greater than $15 \mathrm{~dB}$, which suffices most transmission requirements [20].

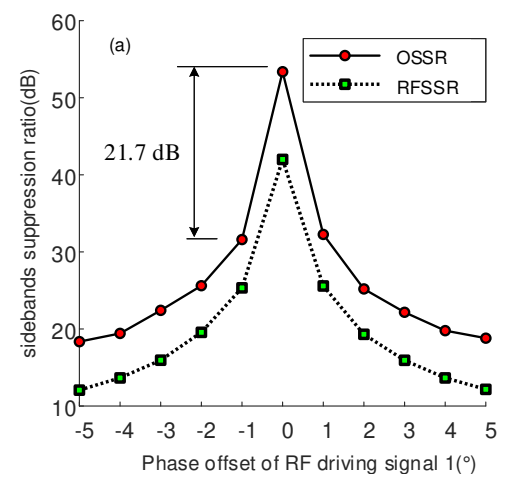

Fig. 7: effect on the OSSR and RFSSR by the offset of RF driving signal phase

3.2 effect on the OSSR and RFSSR by the offset of modulation index

When the modulation index of PolM deviates from the theoretical value, the OSSR and RFSSR decrease sharply. Fig. 8 is the spectral figure measured at point A and Point $\mathrm{C}$ when the modulation index deviates by 0.0314. As the modulation index deviates from the theoretical value, the suppression of sub $\mathrm{A}$ on the carrier component decreases. There is a component of $-32 \mathrm{dBm}$ located at the center carrier, as shown in Fig. 8(a). Then the output optical signal enters into Sub B. Due to the nonlinear effect, the power of the 8th-order optical sideband component in Eq. (6) increases. The optical signal at Point C is shown in Fig. 8(b).

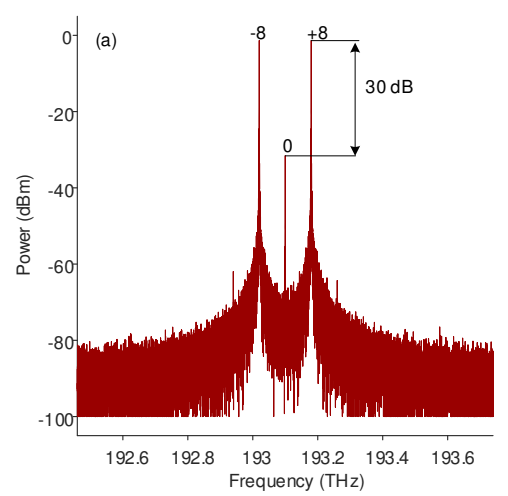

(a)

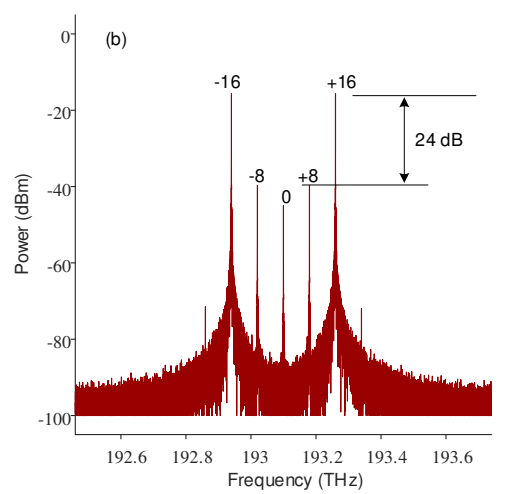

(b)

Fig. 8: Optical Spectrum when the modulation index deviation is 0.0314 . 
When the modulation index's value deviates from the theoretical value, OSSR and RFSSR of the optical Sub-Terahertz wave decrease sharply. From Fig. 9, when the modulation index deviates from theoretical value by $1.4 \%$, OSSR drops from 53 $\mathrm{dB}$ to $12 \mathrm{~dB}$, and RFSSR drops from $42 \mathrm{~dB}$ to $6 \mathrm{~dB}$. When the modulation index deviates from theoretical value, the carrier component of the output signal from Sub A will be poorly restrained, which results in increased harmonic sidebands power and decreased suppression ratio.

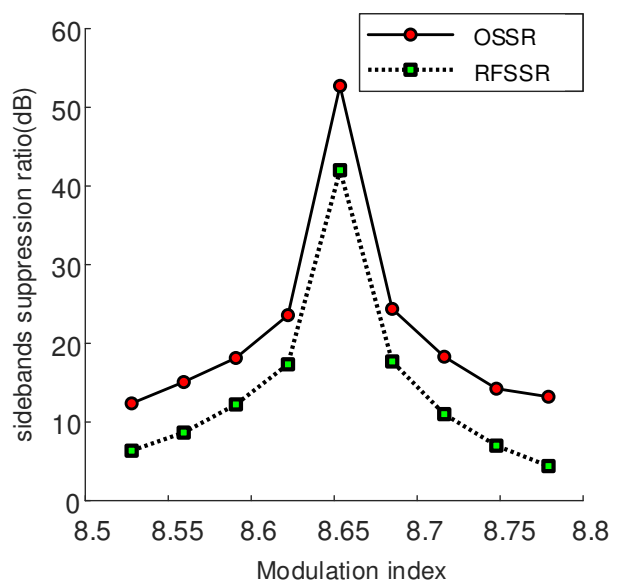

Fig. 9: Effect on the OSSR and RFSSR by the offset of modulation index

\section{3 effect on the OSSR and RFSSR by attenuation deviation}

According to Eq. (10), when the attenuator's attenuation deviates from 15.985 $\mathrm{dB}$, the optical carrier component in Fig. 4(b) will not be completely canceled, resulting in the Sub-THz wave quality deteriorating. Fig. 10 shows the effect on OSSR and RFSSR by attenuation deviation, where OSSR is the suppression ratio between \pm 16 th-order optical sidebands and optical carrier component. As shown in Fig. 10, OSSR decreases gradually with deviation value. When the deviation value is above $0.28 \%$, the RFSSR of the Sub-THz wave decreases with the increase of the deviation value. This is because when the deviation value increases, the optical carrier component power of the system will also increase, causing the clutter power at the $160 \mathrm{GHz}$ frequency after beaten in PD to be greater, which becomes the main factor affecting the Sub-THz wave RFSSR. When the deviation value is within $0.28 \%$, the suppression ratio of \pm 16 th-order optical sidebands to the optical carrier component is above $40 \mathrm{~dB}$. The optical carrier component is not the main factor affecting the quality of submillimeter wave. Therefore, when the deviation value of the optical attenuator is less than $0.28 \%$, the optical carrier component will be well suppressed; when the deviation value is less than $5 \%$, the RFSSR will be above $15 \mathrm{~dB}$, meeting the requirements of the general transmission system. 


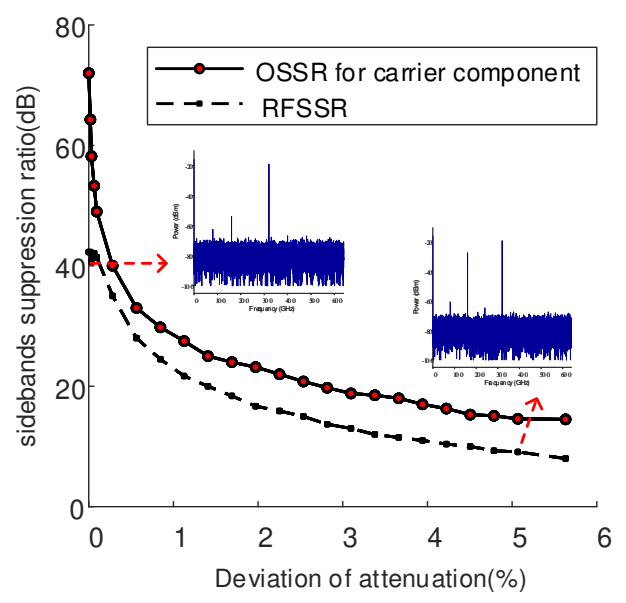

Fig. 10: Effect on OSSR and RFSSR by attenuation deviation

\section{Conclusion}

A novel scheme to generate 32-tupling Sub-THz wave signals by PolM in cascade is proposed. The system consists of Sub A and Sub B, and each subsystem consists of 4 paralleled PolMs. The Sub A is used to pick out \pm 8 nth-order optical sidebands. The Sub B is used to pick out $16^{\text {th }}$-order optical sidebands. An optical phase shifter and an adjustable attenuator are used to cancel the optical carrier component. Through the $\pm 8 \mathrm{n}^{\text {th }}$ - order optical sideband beaten in PD, the frequency 32-tupling $\mathrm{Sub}-\mathrm{THz}$ wave is obtained. According to the scheme proposed, a simulation system is built by OptiSystem, and 32-tupling Sub-THz wave is obtained. The OSSR of the optical signal and RFSSR of the generated 32-tupling Sub-THz are 53dB and $42 \mathrm{dBwhich}$ are consistent with the theoretical value and verify our proposed scheme's feasibility. How the RF drive signal phase and the modulation index of PolM in the system affect the purity of the 32-tupling Sub- $\mathrm{THz}$ wave are also analyzed. We get the conclusion that when the phase deviation is within $\pm 3^{\circ}$, the modulation index deviation is within 0.04, and the attenuator's attenuation deviation is within $5 \%$, the transmission demand can be satisfied.

The proposed scheme does not require an optical filter or DC bias circuit, has a flexible adjustment range, simple structure, and high purity of the generated Sub-Terahertz wave signal. It has greatly potential application in the $\mathrm{THz}$ wave application. Since multiple polarization modulators are used in the scheme, fewer modulators will be considered in subsequent studies to achieve higher frequency Sub-THz wave.

Acknowledgements This work was supported in part by the National Key Research and Development Project (No. 2018YFB1404101). 


\section{References}

1. Shams H, Fice MJ, Gonzalez-Guerrero L, Renaud CC, van Dijk F, Seeds AJ (2016) Sub-thz wireless over fiber for frequency band 220-280 ghz. J Lightwave Technol 34(20):4786-4793

2. Petrov V, Kurner T, Hosako I (2020) Ieee 802.15.3d: First standardization efforts for sub-terahertz band communications toward $6 \mathrm{~g}$. IEEE Commun Mag $58(11): 28-33$

3. Wang D, Tang X, Xi L, Zhang X, Fan Y (2019) A filterless scheme of generating frequency 16-tupling millimeter-wave based on only two mzms. Opt Laser Technol 116:7-12

4. Li W, Yao J (2010) Investigation of photonically assisted microwave frequency multiplication based on external modulation. IEEE Trans Microwave Theory Tech 58(11):3259-3268

5. Qi G, Yao J, Seregelyi J, Paquet S, Belisle C (2005) Generation and distribution of a wide-band continuously tunable millimeter-wave signal with an optical external modulation technique. IEEE Trans Microwave Theory Tech 53(10):3090-3097

6. Jia Z, Yu J, Hsueh YT, Chowdhury A, Chien HC, Buck JA, Chang GK (2008) Multiband signal generation and dispersion-tolerant transmission based on photonic frequency tripling technology for 60 -ghz radio-over-fiber systems. IEEE Photonics Technol Lett 20(17):1470-1472

7. Shih PT, Chen J, Lin CT, Jiang WJ, Huang HS, Peng PC, Chi S (2010) Optical millimeter-wave signal generation via frequency 12-tupling. J Lightwave Technol 28(1):71-78

8. Li Z, Li M, Chi H, Zhang X, Yao J (2011) Photonic generation of phase-coded millimeter-wave signal with large frequency tunability using a polarizationmaintaining fiber bragg grating. IEEE Microw Wirel Compon Lett 21(12):694696

9. Li X, Zhao S, Zhu Z, Gong B, Chu X, Li Y, Zhao J, Liu Y (2015) An optical millimeter-wave generation scheme based on two parallel dualparallel mach-zehnder modulators and polarization multiplexing. J Mod Opt 62(18):1502-1509

10. Baskaran M, Prabakaran R (2018) Optical millimeter wave signal generation with frequency 16-tupling using cascaded mzms and no optical filtering for radio over fiber system. Journal of the European Optical Society-Rapid Publications 14(1):13

11. Wang D, Xi L, Tang X, Zhang X, Zhang W, Tu M, Ding M, Zhao J, Jiang $\mathrm{X}$ (2020) Photonic filterless scheme to generate v-band ofdm vector mm-wave signal without precoding. Opt Commun 466:125663

12. Wang D (2020) V-band vector mm-wave signal generation enabled by a dualparallel mach-zehnder modulator without precoding and optical filter. Microw Opt Technol Lett 62(11):3412-3418

13. Wang D, Xi L, Tang X, Zhang X, Gao N (2020) A simple photonic precodingless scheme for vector millimeter-wave signal generation based on a single phase modulator. Results Phys 19:103412

14. Baskaran M, Prabakaran R, Gayathri T (2019) Photonic generation of frequency 16-tupling millimeter wave signal using polarization property without an optical filter. Optik 184:348-355 
15. Zhu Z, Zhao S, Tan Q, Liang D, Li X, Qu K (2016) Photonically assisted microwave signal generation based on two cascaded polarization modulators with a tunable multiplication factor. IEEE Trans Microwave Theory Tech 64(11):3748-3756

16. Abouelez AE (2020) Photonic generation of millimeter-wave signal through frequency 12-tupling using two cascaded dual-parallel polarization modulators. Opt Quantum Electron 52(3):1-20

17. Kumar A, Priye V (2016) Photonic generation of high frequency millimeterwave and transmission over optical fiber. Appl Opt 55(22):5830-5839

18. Banerjee Chaudhuri R, Das Barman A, Bogoni A (2020) Photonic 60 ghz subbands generation with 24-tupled frequency multiplication using cascaded dual parallel polarization modulators. Opt Fiber Technol 58:102244

19. Wu Z, Cao C, Zeng X, Feng Z, Shen J, Yan X, Wang B, Su X (2020) Filterless radio-over-fiber system based on polarization multiplexing to generate an 80 ghz millimeter wave. Appl Opt 59(24):7455-7461

20. K EM, A SR, G S (2017) Frequency16-tupled optical millimeter wave generation using dual cascaded mzms and 2.5 gbps rof transmission. Optik 140:338346 